\title{
Nickel-Catalyzed Enantioselective Hydroamination of Branched 1,3-Dienes
}

\section{Key words}

nickel catalysis

hydroamination

1,3-dienes

\section{Selected examples:}<smiles>C=C(c1ccc(OC)cc1)C(C)Nc1ccccc1</smiles>

$79 \%$ yield, $r r=12: 1,90 \%$ ee<smiles>C=C(c1ccc(C(F)(F)F)cc1)C(C)Nc1ccccc1</smiles>

$77 \%$ yield, $r r=6: 1,95 \%$ ee<smiles>C=C(c1ccc(OC)nc1)C(C)NCc1ccccc1</smiles>

$78 \%$ yield, $r r=5: 1,95 \%$ ee

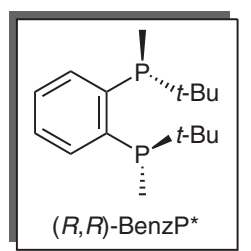

$(R, R)$-BenzP*<smiles>C=C(c1ccc(-c2ccccc2)cc1)C(C)NCc1cccnc1</smiles>

$81 \%$ yield, $r r=7: 1,93 \%$ ee<smiles>C=C(c1ccc(-c2ccccc2)cc1)C(C)NC1CCCC1</smiles>

$76 \%$ yield, $r r=4: 1,92 \%$ ee<smiles>C=C(c1ccc(-c2ccccc2)cc1)C(C)NC(C)c1ccccc1</smiles><smiles>C=C(c1ccc(-c2ccccc2)cc1)C(C)NCCCN</smiles>

$86 \%$ yield, $r r=9: 1,96 \%$ ee<smiles>C=C(c1ccc(-c2ccccc2)cc1)C(C)N1CCCC1</smiles>

$60 \%$ yield, $r r=3: 1, d r=19: 1 \quad 64 \%$ yield, $r r=1.9: 1,95 \%$ ee

$\mathrm{Ni}(\operatorname{cod})_{2}(5 \mathrm{~mol} \%)$

$(R, R)$-BenzP* $(6 \mathrm{~mol} \%)$ $\underset{\text { mesitylene }(0.5 \mathrm{M}), 23^{\circ} \mathrm{C}, 72 \mathrm{~h}}{\stackrel{\text { trifluoroethanol (1.0 equiv) }}{\longrightarrow}}$<smiles>C=C(Br)C(C)NCCC</smiles>

$>99 \%$ recovery, $93 \%$ ee<smiles>C=C(Br)C(C)NCCC</smiles>
not observed

(93\% ee)

Deuterium incorporation studies:<smiles>C=CC(=C)c1ccc(-c2ccccc2)cc1</smiles>

\section{D incorporation \\ $\begin{array}{ll}\text { C4 } & 57 \% \\ & 58 \%\end{array}$}

Significance: The authors report an enantioselective hydroamination of 1,3-dienes by using nickel catalysis. The products were obtained in moderate to good yields and with high enantioselectivities. Reaction monitoring, deuterium labelling, and kinetic studies were performed to elucidate the reaction mechanism.
Comment: Mechanistic experiments reveal the irreversibility of this reaction. Deuterium incorporation was observed in the hydroaminated product when $d_{2}$-benzylamine and $d_{1}$-TFE were employed. Kinetic studies suggest that a $\mathrm{Ni}-\pi$-allyl species arising from hydronickelation is the catalyst resting state. 